\title{
Quality-improvement interventions for osteoporosis: When are the results worth the effort?
}

\author{
Sumit R. Majumdar MD MPH
}

See related research article by Leslie and colleagues on page 290 and at www.cmaj.ca/lookup/doi/10.1503/cmaj.111158

$\mathrm{O}$ steoporosis is a common, chronic and costly disease that affects millions of Canadians and costs billions of dollars each year. ${ }^{1}$ Although the only clinical consequence of osteoporosis is low-trauma fragility fracture, such fractures can cause substantial pain, disability, deformity, diminished quality of life and even death.

Older patients ( 50 years) with low bone mineral density and recent fractures are at high risk of future fractures, which is why secondary prevention is a main focus of the 2010 Osteoporosis Canada guidelines.

Although it has been suggested that older patients with classic osteoporotic fractures can be empirically treated for osteoporosis without testing bone mineral density, such testing remains necessary. Almost all randomized trials for the treatment of osteoporosis include only those patients with low bone density or low bone density and fractures.$^{1-3}$ In addition, bone mineral density testing can be used to establish a baseline or to monitor the response and adherence to treatment. Furthermore, $20 \%-30 \%$ of older patients with fractures have normal bone mineral density and do not require years of preventive medications. $^{2}$

All older patients with fractures should be advised to take adequate amounts of calcium and vitamin $\mathrm{D}$ each day, via supplement and/or diet, and be asked about osteoporosis risk factors and repeated injurious falls. ${ }^{1}$ If a patient has low bone mass (e.g., T-score $<-1.0$ to -1.5 at any site), they should be offered treatment with bisphosphonate drugs. ${ }^{2,3}$ Systematic reviews of bisphosphonate drugs suggest a relative reduction in fracture of about 50\% compared with placebo, as well as a small reduction in all-cause mortality. ${ }^{3}$ When bisphosphonate drugs are contraindicated (e.g., patients with esophagitis, recent dental implants or chronic kidney disease) or not tolerated (e.g., patients with refractory nausea or dyspepsia), several other evidence-based options exist.

Secondary prevention among older patients who present with a fragility fracture is evidence- based and fairly straightforward. Thus, it should be associated with a very high quality of care. However, systematic reviews of studies from many countries suggest rates of bone mineral density testing or treatment for osteoporosis of less than $10 \%-20 \%$ in the 6-12 months postfracture. ${ }^{1}$ Thus, there remain many barriers to delivering optimal postfracture care (Appendix 1, available at www.cmaj.ca/lookup/suppl/doi:10 $.1503 / \mathrm{cmaj} .111999 /$-/DC1). These barriers exist at multiple levels, whether they reflect a disconnect between health care professionals delivering acute care and those responsible for ongoing preventive care, a lack of time on the part of the physician or a lack of knowledge on the part of the patient. ${ }^{4}$ Indeed, the literature reports many studies on gaps and barriers to optimal care for osteoporosis; perhaps, rather than attempting to more precisely describe the problems and catalogue every reason for their existence, it is time we solve them.

Thus, the study by Leslie and colleagues is a welcome addition to the shallow pool of evidence we have for accelerating osteoporosis knowledge translation and improving the quality of postfracture care. ${ }^{5}$ Patients were randomized to one of three groups. Patients in group 1 received usual care, patients in group 2 had mailed notification of the fracture sent to their physicians, and group 3 had notifications sent to both

\section{KEY POINTS}

- Secondary prevention of osteoporosis among older patients who present with a fragility fracture should be associated with a very high quality of care, but only $10 \%-20 \%$ of patients receive optimal management of their osteoporosis in the 6-12 months postfracture.

- There are many barriers to optimal care at the level of the patient, physician and health care system.

- Although the interventions reported by Leslie and colleagues showed only modest improvements over usual care, the effects are clinically worthwhile (number needed to treat $=7$ ).

- As with drugs and devices, it is important to rigorously test qualityimprovement interventions before their wide-scale adoption; in this instance, the interventions tested by Leslie and colleagues seem ready for prime time. 
physicians and patients. The outcomes of the trial were not fractures, but process-of-care measures: the start of treatment for osteoporosis, bone mineral density testing and the composite of testing or treatment within 12 months of the fracture. Such quality indicators have been widely endorsed and, when coupled with achievable benchmarks, are suitable for audit and feedback or pay-for-performance. ${ }^{6}$ Moreover, when process-of-care measures are evidence-based and tightly linked to clinical events, they are sensitive measures of quality and are associated with better outcomes. ${ }^{6}$

This trial is noteworthy because neither physicians nor patients provided informed consent and because both eligibility and outcomes were determined using routinely collected data housed in provincial databases. The province's ministry of health randomized the participants and concealed their allocation from researchers. This is exactly the type of quality-improvement research that fulfills the criteria for waiving informed consent (i.e., low-risk intervention, all treatments available outside the trial, patient identity concealed, outcomes derived from routinely collected data) ${ }^{7}$ the researchers and Manitoba Health are to be lauded for their forward-thinking partnership efforts. In many other jurisdictions, this trial would have automatically required informed consent from both patients and physicians. Ironically, it is entirely permissible for physicians or policymakers to introduce any intervention desired in an uncontrolled fashion without consent and simply assume better outcomes.

Three important results can be highlighted from this trial. First, even in 2010, usual care is disconcertingly suboptimal (3.9\% of patients in this group received bone mineral density testing, and $10.6 \%$ of patients received pharmacologic treatment for osteoporosis). ${ }^{5}$ Second, the combination of physician and patient notification postfracture did not improve outcomes any more than notifying the physician alone. ${ }^{5}$ Third, compared with usual care, the combined interventions led to an absolute increase of $13.4 \%$ over usual care for bone mineral density testing and $14.9 \%$ for the composite end point at a cost of about four or five dollars per patient. ${ }^{5}$

Is a modest improvement in the quality of care worth this amount of effort and research? A meta-analysis of eight trials of postfracture interventions showed a pooled increase of $36 \%$ (95\% confidence interval $[\mathrm{CI}] 21-50)$ in bone mineral density testing and $20 \%$ (95\% CI 10-30) in treatment for osteoporosis, but most of the interventions tested were more complex, expensive and difficult to implement than the interventions reported by Leslie and colleagues. ${ }^{8}$ For example, the use of fracture liaison services or case managers in randomized trials has led to rates of almost $80 \%$ for bone mineral density testing and $50 \%$ for treatment. ${ }^{8}$ In uncontrolled studies, rates of testing or treatment approach $90 \%-95 \%{ }^{4}$ Although the interventions tested by Leslie and colleagues showed improvement over usual care, most of the patients were still not tested or did not receive treatment for osteoporosis. The investigators' a priori minimal important difference was only a $10 \%$ improvement, an arbitrary but common value in the literature. ${ }^{5}$ Ideally, the minimal important difference should be determined before a trial using more validated and transparent methods. For example, osteoporosis experts have previously suggested a $20 \%$ improvement as their minimal important difference, ${ }^{2}$ whereas researchers posited smaller improvements as being worthwhile when interventions are evidence-based, low-risk and low-cost.

Leslie and coworkers have developed and tested an effective, pragmatic, scalable, low-risk and inexpensive intervention for patients scattered over large geographic areas. Although the effects are modest, they are clinically worthwhile. Their interventions could be easily adopted elsewhere; not only should they be the new usual care in Manitoba, they should set the standard for control arms of future studies of quality improvement in osteoporosis.

\section{References}

1. Papaioannou A, Morin S, Cheung AM, et al. 2010 clinical practice guidelines for the diagnosis and management of osteoporosis in Canada: summary. CMAJ 2010;182:1864-73.

2. Majumdar SR, Johnson JA, McAlister FA, et al. Multifaceted intervention to improve diagnosis and treatment of osteoporosis in patients with recent wrist fracture: a randomized controlled trial. CMAJ 2008;178:569-75.

3. Bolland MJ, Grey AB, Gamble GD, et al. Effect of osteoporosis treatment on mortality: a meta-analysis. J Clin Endocrinol Metab 2010; 95:1174-81

4. Majumdar SR. A T-2 translational research perspective on interventions to improve post-fracture osteoporosis care. Osteoporos Int 2011;22(suppl 3):471-6.

5. Leslie WD, LaBine L, Klassen P, et al. Closing the gap in postfracture care at the population level: a randomized controlled trial. CMAJ 2012;184:290-6.

6. Higashi T, Shekelle PG, Adams JL, et al. Quality of care is associated with survival in vulnerable older patients. Ann Intern Med 2005;143:274-81.

7. Miller FG, Emanuel EJ. Quality-improvement research and informed consent. N Engl J Med 2008;358:765-7.

8. Little EA, Eccles MP. A systematic review of the effectiveness of interventions to improve post-fracture investigation and management of patients at risk of osteoporosis. Implement Sci 2010; 5:80-8.

Affiliation: Sumit R. Majumdar is with the Department of Medicine, University of Alberta, Edmonton, Alta.

Funding: Sumit R. Majumdar receives a salary support award from Alberta Heritage Foundation for Medical Research and Alberta Innovates - Health Solutions (Health Scholar) 